\title{
Dotando de sentido el algoritmo de la división a partir de una trayectoria de aprendizaje que parte de lo concreto para llegar a lo simbólico 5
}

Sense giving the division algorithm from a learning path part of the concrete to reach the symbolic

Sense dando o algoritmo da divisão de uma parte do caminho
de aprendizagem do concreto para chegar ao simbólico

Recibido: mayo de 2013

Aceptado: agosto de 2013
Carlos Alberto Díez Fonnegra ${ }^{6}$

Oscar Leonardo Pantano Mogollón ${ }^{7}$

Leidy Viviana Pantano Mogollón ${ }^{8}$

\section{Resumen}

El taller titulado dotando de sentido el algoritmo de la división a partir de una trayectoria de aprendizaje que parte de lo concreto para llegar a lo simbólico pretende enseñar esta propuesta de innovación que se ha construido en el proyecto "Matemáticas para la vida" de la Fundación para el Desarrollo Educativo y Pedagógico. Esta propuesta ha sido diseñada con el propósito de dotar de sentido el aprendizaje del algoritmo de la división, evidenciando la lógica en que se sustenta, además de la correcta aplicación de los diferentes pasos que lo componen. Lo anterior se realiza a través de la utilización de material concreto y la delimitación de una trayectoria de aprendizaje.

Palabras clave: Matemáticas escolares; números; operaciones aritméticas; división; algoritmo de la división; material concreto; enseñanza; planificación del profesor; metodología de enseñanza; metodología de trabajo en el aula; innovación; pensamiento numérico; pensamiento geométrico.

\begin{abstract}
The workshop entitled sense giving the division algorithm based on a learning path part of the concrete to reach the symbolic attempt to teach this innovative proposal that has been built in the project "Mathematics for Life" Foundation for Educational Development and Teaching. This proposal has been designed with the purpose of learning to make sense of the division algorithm, showing logic underpinning, as well as the correct application of the different steps that comprise it. This is done through the use of specific material and the delimitation of a learning path.
\end{abstract}

Keywords: school mathematics; numbers, arithmetic operations, division, long division, concrete material, teaching, teacher planning, teaching

5 Artículo de Investigación

6 Fundación para el desarrollo educativo y pedagógico. Colombia. Contacto: carlosd@fundacionedp.org;

7 Fundación para el desarrollo educativo y pedagógico. Colombia. Contacto: leonardop@fundacionedp.org

8 Universidad Distrital Francisco José de Caldas. Bogotá, Colombia. Contacto: lvpmogollon@gmail.com 
methodology, methodology of work in the classroom innovation thinking numeric geometric thinking.

\section{Resumo}

O workshop intitulado sentido dar o algoritmo da divisão com base em uma parte do caminho de aprendizagem do concreto para chegar a tentativa simbólica para ensinar esta proposta inovadora que foi construída no projeto "Matemática para a Vida" da Fundação para o Desenvolvimento da Educação e Ensino. Esta proposta foi concebida com o propósito de aprender a fazer sentido do algoritmo da divisão, mostrando esteio lógica, bem como a correcta aplicação das diferentes etapas que o compõem. Isto é feito através da utilização de material específico e a delimitação de um caminho de aprendizagem.s.

Palavras-chave: matemática da escola, números, operações aritméticas, a divisão, a divisão longa, material concreto, ensino, planejamento de professores, metodologia de ensino, metodologia de trabalho na inovação em sala de aula pensando pensamento geométrico.

\section{Introducción}

El propósito fundamental del taller dotando de sentido el algoritmo de la división a partir de una trayectoria de aprendizaje que parte de lo concreto para llegar a lo simbólico pretende enseñar a los participantes la propuesta de innovación para la enseñanza del algoritmo de la división en el CICLO DE EDUCACIÓN BÁSICA A, más específicamente para los grados tercero y cuarto de primaria. Además, el taller pretende promover la implementación de esta propuesta en el quehacer pedagógico de los participantes, con el propósito de ayudar a sus estudiantes en el aprendizaje del algoritmo de la división.

Esta propuesta ha sido diseñada de tal forma que permita dotar de sentido los diferentes procedimientos o reglas que se realizan en el algoritmo de la división y que usualmente no suelen ser tan explícitas en la enseñanza de éste, y por lo tanto, no permiten que se adquiera una sólida comprensión de lo que se hace. De este modo, la propuesta posibilita que los estudiantes, a través del material concreto y la manipulación de éste, observen las diferentes reglas que organizan o secuencian el algoritmo.
Además, la propuesta cuenta con la delimitación de una ruta o trayectoria de aprendizaje, que parte de lo concreto para llegar a lo simbólico, es decir, se ha diseñado teniendo en cuenta una transición entre: el lenguaje natural y la manipulación del material, la utilización del lenguaje simbólico ligado al material y así finalmente, emplear el lenguaje simbólico sin la ayuda del referente concreto. De este modo, se han definido una serie de actividades que posibilitan que a medida que se avanza en el proceso se vaya complejizando y acercando el lenguaje matemático disciplinar utilizado por la comunidad matemática.

Por otro lado, la vinculación y utilización de material concreto se realiza con el propósito de establecer una relación entre el algoritmo de la división y el pensamiento geométrico, promoviendo que los estudiantes construyan una imagen mental del algoritmo, relacionada con la construcción de rectángulos, es decir, asocien a cada regla o paso que se realice en el algoritmo una actividad manipulativa con el material. En este sentido, permite que las partes constitutivas del algoritmo (dividendo, divisor, cociente y residuo) estén asociadas respectivamente al área de un rectángulo, su altura, su base y las piezas sobrantes cuando la división no es exacta. Está asociación posibilita que los 
estudiantes construyan un referente para cada una de estas partes, dotándolas de sentido.

El desarrollo del taller estará orientado por una serie de actividades que permitan evidenciar a los participantes la trayectoria de aprendizaje establecida, la manera en que se puede utilizar el material y los diferentes niveles de abstracción que están establecido en el proceso gradual de aprendizaje que parte de lo concreto para llegar a lo simbólico. Lo anterior, con el propósito de que puedan reconocer como el material puede ser utilizado para construir el algoritmo de la división.

\section{Marco teórico.}

La enseñanza del algoritmo de la división suele centrarse en la adquisición y dominio de la secuencia de reglas que deben efectuarse una y otra vez para obtener el resultado, privilegiando la adquisición de la destreza para resolver divisiones más que la comprensión del proceso y de las diferentes reglas que rigen éste y que están en estrecha relación con el sistema de numeración base diez.

Como afirman Gairín \& Rocher (2002); Roa (2001) lo más frecuente en la enseñanza de los algoritmos es que se dé prioridad a la ejecución correcta de la secuencia de pasos que lo componen y que están encaminadas a la obtención del resultado, provocando que prime la ejercitación frente a la comprensión. Por esta razón, es indispensable promover el descubrimiento de las reglas que rigen o sustentan los algoritmos y que usualmente suelen estar ocultas en estos.

Por otro lado, en la enseñanza del algoritmo de la división suelen concebirse todas las cifras que aparecen allí como unidades, sin tener en cuenta que cada una de ellas representa una unidad de orden superior diferente, debido al valor posicional en el que se encuentran, ocasionando que no se reconozcan la influencia que tiene el sistema de numeración base diez en el algoritmo.

El algoritmo de la división se diferencia de los demás, dado que en éste no se disponen los números uno debajo de otro, ni se opera de derecha a izquierda, además, el resultado obtenido no necesariamente es un único número sino dos: el cociente y el residuo. En este algoritmo es indispensable reconocer que en el dividendo las cifras van cambiando de valor posicional a medida que se van efectuando las diferentes reglas que lo sustentan.

Como afirma Roa (2001) en la realización del algoritmo de la división es indispensable en cada momento reconocer de qué tipo son las unidades que se están operando, de cuántas unidades de un determinado orden se van a utilizar cuando no se tengan las necesarias del orden inmediatamente superior para repartir y, de garantizar que se repartan siempre la mayor cantidad de unidades con el propósito de que las unidades sobrantes sean menores en cantidad que el divisor. Estos aspectos son poco reconocidos por los estudiantes, dado que la atención se centra en la correcta aplicación de los pasos y no en las reglas ocultas asociadas al sistema de numeración base diez.

Por esta razón, para una mayor comprensión del algoritmo y de las reglas ocultas en que se sustenta éste, es indispensable establecer una trayectoria de aprendizaje que permita por un lado evidenciar esas reglas ocultas que están estrechamente relacionadas con el funcionamiento de los algoritmos y por otro los conocimientos matemáticos implícitos en éste como el sistema de numeración base diez, la desagrupación de unidades de orden superior en unidades de orden inmediatamente anterior. Para ello se requiere realizar una transposición didáctica de este objeto matemático procedimental, que exige pasar del saber sabio, al saber enseñar, es decir, del conocimiento matemático formal que se ha construido a lo largo de la historia en conocimiento asequible a los estudiantes (D'Amore, 2006).

Castro \& Castro (2011) plantean que: "El concepto de división lleva implícito un proceso de generalización y abstracción progresiva que va de los objetos a los símbolos y de éstos al cálculo numérico" (p. 120). Lo anterior, se puede realizar a través de la utilización de material concreto y la delimitación de una serie de actividades o situaciones problema que permitan partir de la utilización de este material para trabajar posteriormente con los números 
de manera simbólica y manipular dichos símbolos sin necesidad de hacerlo con el material, y así posteriormente centrar la atención en la adquisición de la destreza, es decir en la constante ejercitación para resolver divisiones.

Roa (2001) propone que es indispensable en el aprendizaje de los algoritmos que los estudiantes realicen una gran variedad de actividades que involucren la utilización de material concreto, especialmente aquellos que tenga la misma estructura que el sistema de numeración base diez, debido a que las operaciones y sus diferentes algoritmos están organizados por éste. La importancia de utilizar material concreto se sustenta en que las acciones realizadas con éste posibilitan que los estudiantes las interioricen progresivamente, construyendo imágenes mentales asociadas a los objetos matemáticos que se pretenden enseñar. Estas imágenes mentales permiten que se enriquezca estructuralmente el pensamiento porque lo que se hace de manera simbólica está ligado a un referente concreto que permite dotar de significado lo que se está haciendo.

De la misma manera, Dienes (citado por Resnick \& Ford, 1990) afirma que:

Los niños son constructivistas por naturaleza, más que analíticos. Van formándose (es decir, construyendo) una imagen de la realidad a partir de sus experiencias con los objetos del mundo. Este proceso depende mucho de una exploración activa (...) dado que las relaciones y pautas matemáticas no son evidentes en el entorno diario de los niños. (p. 143)

Teniendo en cuenta los planteamientos expuestos anteriormente que justifican la importancia de utilizar material concreto en el aprendizaje de los objetos matemáticos, en esta propuesta el material se ha utilizado con otra finalidad que consiste en: establecer una relación entre lo que se hace en el algoritmo (pensamiento numérico) y la construcción de rectángulos a partir de su utilización (pensamiento geométrico), permitiendo integrar los ejes del pensamiento matemático, haciendo mucho más enriquecedor el aprendizaje de los estudiantes.
Además, el material utilizado posibilita identificar las diferentes unidades que se están operando a medida que se desarrollan la secuencia de pasos y operaciones aritméticas que organizan el algoritmo de la división, característica que no se ve reflejada o no es totalmente explícita en la enseñanza del algoritmo de manera simbólica.

La utilización de las piezas pretende que los estudiantes construyan rectángulos dada la cantidad de piezas que los conforman (dividendo), que en algunos casos no necesariamente deben utilizarse completamente sino que pueden sobrar algunas (residuo), y la dimensión de la altura que deben tener estos (divisor). A partir de estos elementos, los estudiantes deben encontrar la otra dimensión de la base del rectángulo (cociente). Esta construcción de rectángulos con el material utilizado posibilita que los estudiantes doten de sentido las partes constitutivas del algoritmo (dividendo, divisor, cociente y residuo), dado que están ligadas a un referente concreto y más específicamente a un objeto matemático del pensamiento geométrico, posibilitando así que los estudiantes construyan imágenes mentales mucho más sólidas.

\section{Descripción de las actividades.}

El desarrollo del taller estará orientado por tres momentos esenciales de la siguiente manera:

En el primer momento se explicarán las bases teóricas que sustentan la propuesta, haciendo énfasis en los aspectos didácticos que guían las acciones realizadas, tales como la elección del material, la trayectoria de aprendizaje establecida y las posibles actividades que se pueden desarrollar acorde con esta. A su vez, se dará a conocer cómo es posible construir el material y las condiciones que debe tener el diseño de este para que se ajuste a lo que se pretende realizar.

En el segundo momento se dará inicio a la manipulación del material concreto, con el propósito de que los participantes se familiaricen con este, evidenciando las características que tiene. Además, se pretende que empiecen a pensar en la manera como puede ser utilizado para enseñar el objeto 
matemático establecido, permitiendo que surjan nuevas ideas que pueden complementar la propuesta.

Seguido, se dará inicio al desarrollo de una serie de actividades que permitan evidenciar la trayectoria de aprendizaje establecida para construir el algoritmo de la división, haciéndose explicita la manera cómo debe utilizarse el material concreto y los diferentes conceptos matemáticos inmersos en esta.

Finalmente, en el tercer momento del taller, se realizará una socialización, en la cual se discutirán las actividades realizadas, la pertinencia de estas y del material utilizado y las potencialidades o limitaciones de la propuesta, con el propósito de retomar los aportes realizados por los participantes para consolidarla.

\section{Referencias}

Bruno, D. Didáctica de la matemática. En: Colombia 2006. Ed: Coopertiva Editorial Magisterio

Castro, R. \& Castro, R. (2011). Didáctica de las matemáticas: de preescolar a secundaria. Bogotá: Ecoe Ediciones.

Gairín, M., \& Sancho, J. (2002). Números y algoritmos. España: Editorial Síntesis.

Resnick, L., \& Ford, W. (1990). La enseñanza de las matemáticas y sus fundamentos psicológicos. Barcelona: Ediciones Paidós Ibérica, S.A.

Roa, R. (2001). Algoritmos de cálculo. En E. Castro (Ed.), Didáctica de las matemáticas en la educación primaria (pp. 231-254). España: Editorial Síntesis. 